\title{
Effect of Physical Activity/Exercise Interventions on Immune Parameters, and Inflammatory Markers for Proxy Conditions Among Adults Prone to COVID-19: A Systematic Review Protocol
}

Ebuka Miracle Anieto ( $\square$ antebu001@myuct.ac.za )

Department of Health and Rehabilitation Sciences, University of Cape Town, South Africa https://orcid.org/0000-0002-8160-9701

Veronica Ebere Ogbodo

University College Hospital Ibadan

ljeoma Blessing Nwadilibe

University of Cape Town Faculty of Health Sciences

Omotoyosi Johnson Adu

University College Hospital Ibadan

Bouwien C.M. Smits-Engelsman

University of Cape Town, Department of Health and Rehabilitation Sciences, Faculty of Health Sciences Jaleel Mohammed

Rehabilitation Association for Hematopoetic Cell Transplant, UK

Michael Ebe Kalu

McMaster University School of Rehabilitation Sciences

\section{Protocol}

Keywords: COVID-19, SARS-CoV-2, Systematic review, Meta-analysis, Exercise, Physical activity

Posted Date: August 19th, 2020

DOI: https://doi.org/10.21203/rs.3.rs-60112/v1

License: (c) (i) This work is licensed under a Creative Commons Attribution 4.0 International License.

Read Full License 


\section{Abstract}

\section{Background}

Older individuals (over 60 years) with hypertension, diabetes, cardiovascular disease, chronic respiratory disease, and cancer are at the highest risk of contracting and dying from Coronavirus (COVID-19). Compromised immunity (both innate and adaptive) and increased inflammatory response (cytokinestorm syndrome) are predictors for high mortality among this population group. Exercise/physical activity seems to be a plausible way to decrease both the risk of transmission and mortality, and improve health outcomes among this population since there is no available treatment for COVID-19. The study will investigate the effectiveness of physical activity/exercise in improving the immune parameters and reducing the inflammatory biomarkers in proxy conditions that make individuals susceptible to COVID-19.

\section{Methods}

The Preferred Reporting Items for systematic reviews and Meta-Analyses Protocol (PRISMA-P) 2015 will guide this review. We will search ten databases (until August 2020) to include randomized control trials articles that explored the effectiveness of physical activity/exercise in improving immune parameters and reducing inflammatory biomarkers in proxy conditions (hypertension, diabetes, cardiovascular disease, chronic respiratory disease and cancer). Two review authors will independently screen citations (title and abstract), extract data (using standardized forms), assess the risk of bias (using Cochrane risks of bias) and quality of data (using GRADE). Homogenous studies will be analyzed using the fixed-effect model of meta-analysis, while a narrative synthesis will be conducted for heterogeneous studies.

\section{Discussion}

There are no specific physical activity/exercise parameters (frequency, intensity, type of exercise and time- FITT) for interventionists to use when developing high-quality RCT for individuals vulnerable to COVID-19. Therefore, it is important to review the literature to identify and highlight the exercise FITT parameters that increase the immune outcomes and reduce inflammatory biomarkers for proxy conditions that make individuals susceptible to COVID-19. It is also important to identify the specific exercise regimen suitable and beneficial for each proxy group.

\section{Systematic review registration}

PROSPERO CRD42020196907

\section{Background}

The Severe Acute Respiratory Syndrome Coronavirus 2 (SARS-CoV-2) or COVID-19, as was named by the World Health Organization (WHO) [1], is a novel coronavirus that has resulted in a pandemic. The first record of the disease was on 31 December 2019 in Wuhan, a city in Mainland China [2] and is currently rapidly spreading to the rest of the world. Owing to its rate of spread and fatality with a case fatality rate 
estimated at 2-5\% [3], the WHO pronounced the outbreak a global health emergency on 30 January 2020. As of 14 August 2020, 10:42 AM Central European Summer Time, 20,687,815 cases and 750,400 deaths have been reported globally [4]. Among other reported symptoms resulting from this virus, respiratory disorders such as respiratory failure, acute respiratory distress syndrome, and pneumonia are significant symptoms associated with fatality $[5,6,7]$. In moderate to severe cases, COVID-19 attacks the respiratory system $[8,9]$, which progresses to a respiratory failure resulting from a damage to the alveoli, which could be fatal or leave the patients not only in critical need for supplemental oxygen and mechanical ventilator but also with residual lung damage that may require extended recovery time [10].

Globally, the mortality rate as a result of COVID-19 is on a steady increase. Although all population groups are at risk of contracting COVID-19, some individuals with specific comorbidities are more susceptible than others. A meta-analysis of 6 studies involving 1527 confirmed cases reported that hypertension (17.1\%), cardio-cerebrovascular conditions (16.4\%), and diabetes mellitus (9.7\%) were the most prevalent cardiovascular and metabolic conditions associated with COVID-19 [11]. Zeng et al. [12], Paroh et al. [13] and Tian et al. [14] conducted a systematic review and included 13, 14 and 14 studies, respectively. The studies reported that men aged 60 years and above with conditions such as hypertension, diabetes, cancer, cardiovascular and respiratory diseases were at higher risk of contracting, developing serious symptoms and dying from COVID-19.

Similarly, Jain and Yuan [15] reviewed seven articles that evaluated the characteristics of COVID-19 patients admitted in an intensive care unit and reported that patients with chronic obstructive pulmonary disease (COPD) were the most vulnerable, followed by patients with cardiovascular disease and hypertension. Furthermore, the World Health Organization has also identified hypertension, cardiovascular disease, diabetes, chronic respiratory disease such as COPD, and cancer as risk factors for contracting and developing serious illness from COVID-19 [16]. Summarily, people with comorbidities such as hypertension, diabetes, cardiovascular disease, chronic respiratory disease and cancer have been identified as the high-risk individuals that are the most vulnerable, susceptible to COVID-19 with a high mortality rate [17]. In this study, we refer to these comorbidities as proxy conditions prone to developing COVID-19.

Notably, individuals with these proxy conditions by default have lower immunity, and these make them more susceptible to developing COVID-19. For instance, diabetes mellitus (DM) increases patients' susceptibility to viral infections due to its negative impact on both the innate and adaptive immune parameters $[18,19,20]$. Besides, more than $80 \%$ of individuals with type-2 DM have hypertension [21]. Compromised immunity has also been reported in patients with cancer, which increases their susceptibility to opportunistic infections [22]. Worthy to note, a decrease in adaptive immunity marked by a significant reduction in CD4 and CD8 cells have been reported among individuals with COVID-19 [23, 24]. Similarly, a study reported that there is usually a significant reduction in the total T cells, CD4 and CD8 T cell counts, which is a reliable marker of disease severity in patients with COVID-19 [25]. Recent studies showed that $82.1 \%$ of patients with COVID-19 have decreased circulating lymphocytes (a 
constituent of both innate and adaptive immunity) $[7,26,27]$. The combined impact of the comorbidities and SARS-CoV-2 on immunity results in a very poor clinical outcome in these population groups.

Also, individuals with hypertension, diabetes, cardiovascular (e.g. congestive heart failure) and cardiopulmonary conditions (e.g. COPD) have an increased plasma level of inflammatory biomarkers, for example C-reactive protein (CRP), interleukin-6 (IL-6), and tumor necrosis factor-a (TNF-a) [28, 29, 30, 31, $32,33,34]$. Elevated inflammatory responses have also been reported among patients with cancer. $A$ study reported an increased serum concentration of IL- 6 , IL-1 $\beta$, TNF-a, and CRP in patients with cancer as compared with healthy individuals [35]. Notably, increase in the inflammatory response (cytokine release syndrome), marked by an elevated concentration of inflammatory cytokines (Interleukin-6, Interleukin-10, Interleukin-2, Tumor necrosis factor- $a$, Interferon- $\gamma$ ) is one of the significant causes of disease severity and mortality in patients with COVID-19 [36]. An elevation in inflammatory biomarkers results to an increased vascular permeability, which causes an influx of a large amount of fluid and blood cells into the alveoli, consequently causing dyspnea and respiratory failure [37, 38, 39]. Interestingly, studies have reported that immune parameters, T-cells (CD8 and CD4) counts have a negative correlation with inflammatory markers (IL-6, IL-10, and TNF-a), with COVID-19 disease resolution marked by a reduced concentration of IL-6, IL-10, and TNF-a, and increased T-cell counts [25]. The phenomenon is an indication that the reduction of T-cells noted in patients with COVID-19 could be a consequence of the increased concentration of TNF-a, IL-6, and IL-10, which negatively regulates T-cell survival or proliferation [25].

Exercise improves both innate and adaptive immunity. Aerobic exercise of moderate to high intensity performed for 30 minutes, 3-5 times per week, enhances the antipathogen functions of tissue macrophages with an associated increase in recirculation of immunoglobulins, natural killer cells, cytotoxic T cells, anti-inflammatory cytokines, neutrophils, and immature B cells [40, 41, 42, 43, 44]. All of these immune-markers play critical roles in metabolic health and immune defense activity [40].

Similarly, physical activity/exercise has a negative correlation with inflammatory biomarkers [45]. Several studies have reported that exercises reduce inflammatory biomarkers including the serum concentration of CRP, IL-6, IL-18, TNF-a, and IL-1a, in individuals at risk of cardiovascular diseases $[46,47,48,49,50]$, and type 2 DM [51]. Previous studies have reported that exercise reduces the concentration of CRP and TNF-a among individuals with hypertension [52] and cancer [53], respectively.

Given that there is no treatment for COVID-19, the emphasis is placed on prevention, among other means, improving the body's immunity against the virus is a crucial factor. Exercise is a reliable and most accessible form of prevention strategy; however, the impact of exercise on COVID-19 is still at its testing stage. Since studies have identified comorbidities that predispose individuals to developing COVID-19, we believed that reviewing the impact of exercises on the immune parameters of these proxy conditions is warranted. Because improving the body's immunity and reducing the concentration of inflammatory biomarkers in those identified vulnerable groups would be useful in the preventive approach, thus, reducing the risk of contracting COVID-19, and reducing the risk of mortality due to COVID-19. Besides, this review will provide a comprehensive exercise Frequency-intensity-time-type that interventionists can 
use in developing randomized control trials to test the impact of exercise on the course of the disease, chances of hospitalization, landing on ICU and surviving from COVID-19.

This study aims to investigate the effectiveness of physical activity/exercise in improving the immune parameters and reducing the inflammatory biomarkers in proxy conditions that make individuals susceptible to COVID-19. Specifically, this study will aim to answer two research questions: (a) What are the exercise FITT parameters that increase the immune outcomes and reduce inflammatory biomarkers for proxy conditions that make individuals susceptible to COVID-19?; (b) What are the specific exercise regimen suitable for each of the proxy conditions i.e. what specific exercise regimen is beneficial per proxy group?

\section{Methods}

The Preferred Reporting Items for systematic reviews and Meta-Analyses Protocol (PRISMA-P) 2015 will guide this review [54,55]. This protocol is registered in PROSPERO database (CRD42020196907).

\section{Inclusion criteria}

\section{Studies will be included if:}

1. published in English language.

2. participants were individuals susceptible to COVID-19 infection, specifically people with diabetes, heart diseases, hypertension, cardiovascular, cardiopulmonary diseases and cancer.

3. physical activities or exercise were the interventions. Physical activity is referred to as any bodily movement (both structured and unstructured) involving the skeletal muscles with a consequent consumption of energy [56]. There will be no restrictions bothering on the exercise type, frequency, time, intensity and duration or the period of follow up after the intervention. Studies that consider physical activity/exercise in addition to other interventions such as drugs and nutritional intervention, if we can extract the effect of the physical activity on the selected outcome.

4. studies were carried out in hospitals, outpatient clinics, rehabilitation centers, or community settings.

5. studies were randomized controlled trials (RCTs).

6. studies control were any intervention other than physical activity/exercise. Such interventions may include conventional therapies, usual care, sham intervention or no intervention.

7. studies considered immune parameters or inflammatory biomarkers as outcomes.

- The primary outcomes to be considered in this review are: Immunoglobulin A (IgA), Immunoglobulin G (IgG), Immunoglobulin M (IgM), Immunoglobulin E (IgE), Immunoglobulin D (IgD), CD4 count, CD8 count, CD4/CD8 count, CD19 count, CD20 count and Natural Killer (NK) cells. These outcomes were considered primary because they play major roles in the body's defense mechanism against infections. 
The secondary outcomes are inflammatory biomarkers such as; CRP, IL-6, IL-18, IL-10 TNF-a, and IL-1a, Interferon-y leptin, and resistin. The outcomes will only be considered if they were measured with generic validated tools.

\section{Search strategy}

The search strategy which will be used to identify relevant studies was developed and piloted following the guideline outlined in the Cochrane handbook for systematic reviews of intervention studies [57], as well as the recommendation by the center for review and dissemination [58]. The search strategy was developed in consultation with a health librarian using the Medical Subject Headings (MeSH) and keywords relating to the review title. Search strings of the individual PICO components was built into Boolean expressions using Boolean commands ('OR,' 'AND,' and 'NOT'). See Appendix 1 for an example of the search strategy. This search strategy will be adapted for use in the various databases and further published in the final report as supplementary materials. Studies will be searched for in the following databases: MEDLINE/PubMED, Cochrane Library, Cumulative Index to Nursing and Allied Health Literature (CINAHL), OVID, Allied and Complementary Medicine Database (AMED), ProQuest health and medical complete, PsycINFO, Web of Science Core Collection and Physiotherapy Evidence Database (PEDRO). We will also conduct searches in clinical trial registers and directory of open-access repository websites, which include http://www.clinicaltrial.gov, http://www.opendor.org, as well as the Web of science conference proceedings. In addition, the reference lists of the identified articles will be searched.

\section{Study Selection}

Two independent reviewers will select the studies following the study eligibility criteria. All search citations will be collected, and the duplicate citations will be removed using Refworks ${ }^{\mathrm{TM}}$. Study selection will be conducted in two phases using Covidence (ㄷ 2017 Covidence). First, all titles and abstracts of identified articles will be screened by two reviewers independently. Second, the full-texts of the articles that passed the first stage of the screening will be screened independently. In cases where there are two versions of the same article, the most recent or most comprehensive version of the article will be included. If any inclusion disagreements arise at any stage of data selection, a third reviewer will be consulted. The kappa statistics $(95 \% \mathrm{Cl})$ will be used to test the level of agreement on the selected articles emerging from the independent reviewers. Furthermore, we will contact the authors of studies for a maximum of three email attempts to clarify issues bothering on selection of any study when a conclusion could not be reached based on the information available. If any of the contacted authors did not respond to the emails, the article will be excluded, and the reason for exclusion will be clearly stated. The PRISMA flow chart will be used to track and present the study process.

\section{Risk of Bias assessment}

The potential risk of bias $(\mathrm{ROB})$ in the included studies will be assessed using the Cochrane Collaboration risk of bias tool 2 (ROB 2), used for studies that aim at evaluating the effect of assignment to treatment/intervention (the 'intention-to-treat' effect) [59]. The tool is comprised of five key domains; (1) 
Risk of bias arising from randomization (random sequence generation, allocation of concealment, and baseline differences), (2) deviations from the intended interventions (participants and personnel blinding, deviations from study protocol, appropriate data analysis), (3) Missing outcome data (intention to treat analysis), (4) Risk of bias in outcome measurement (appropriate outcome measure method, assessor blinding), (5) Risk of bias in selection of the results to report (selective outcome reporting). Each domain will be graded as 'high risk', 'low risk', or 'some concerns' for all the included studies. Afterwards, an overall risk of bias judgement will be given as either 'low risk' or 'high risk' based on the ROB in the five domains. In a case where there is insufficient information to decide the potential ROB in any study, the authors will be contacted three times through mail and in the absence of any response or adequate clarification, the potential ROB will be considered as 'unclear.' Two independent reviewers will make the judgment on the potential ROB. Areas of discordance will be addressed by discussion for a consensus and/or consultation with the third reviewer.

\section{Quality assessment}

The Grading of Recommendations Assessment, Development and Evaluation (GRADE) will be used in judging the quality of evidence of the included articles [60] in various domains: risk of bias, consistency, directness, precision, and publication bias. Evidence from the studies will be rated as 'high quality', 'moderate quality', low quality', and 'very low quality [60]. See Appendix 2.

\section{Data collection processes}

A pilot data collection will be carried out using the template proposed by the Cochrane Consumers and Communication Review Group Data Extraction [61]. Subsequently, two reviewers will independently perform data extraction from the included articles using the same tool. Any discordance stemming from the extraction will be tackled through discussion and reflection, in consultation with a third reviewer if necessary.

\section{Data items}

We will extract the following information from the included articles: authors reference, participants' characteristics (which includes age range, gender composition, inclusion and exclusion criteria, diagnosis, disease duration and chronicity), study sample size (both intervention and control groups), study design, intervention (components/parameters, context/mode of delivery, intervention and follow-up duration, follow-up), outcome(s) assessed, the outcome(s) measurement methods, results, and conclusions.

\section{Data synthesis and analysis, including assessment of heterogeneity}

Based on this study aim (stated above), we will evaluate, compare and pool in evidence on the effectiveness of intervention on both the primary and secondary outcomes from the included articles. An overall estimate of effect of the intervention will then be conducted using a meta-analysis to answer the review questions [62] for homogenous outcomes. We will conduct a narrative synthesis of data from heterogeneous studies. 


\section{Statistical analysis}

We will conduct the analysis and presentation of results in order of hierarchy with the primary outcomes coming before the secondary outcomes. There may be a significant heterogeneity coming from variances in the clinical characteristics of participants, diverse proxy conditions to COVID-19 studied, different types of intervention, statistical strategy, and outcome measurement instrument utilized. Thus, we will assess the heterogeneity in included studies using the Cochran's $X^{2}$ test, which will be quantified using $\mathrm{I}^{2}$ [63]. Homogenous studies will be pooled together for meta-analysis using a fixed-effects model [57]. This approach compares standardized mean differences in the intervention and control groups for the included studies $(95 \% \mathrm{Cls})$. Rev Man 5 software will be utilized for the meta-analysis.

\section{Sensitivity analysis}

We shall conduct sensitivity analysis to determine the potential influence on the treatment effect in the event of many included studies with significant heterogeneity in study characteristics. For instance, intervention setting (e.g. hospital vs home/ community based), type of intervention (e.g. aerobic vs strengthening vs resistance exercises), and/or study group (e.g. individuals living with hypertension vs diabetes). This will only be carried out if there are more than two studies, and not less than two of the studies are a homogenous subset.

\section{Publication bias/ Meta-biases}

We will assess meta-bias using the funnel-plot for asymmetry followed by the Egger regression test [63].

\section{Reporting of the review}

We will report the systematic review following the guideline stated in the Preferred Reporting Items for Systematic reviews and Meta-Analyses (PRISMA) Statement [54]. All items relevant to the review will be reported accordingly. A PRISMA checklist will be published with the final report.

\section{Potential amendments}

Considering that quantitative studies are easily influenced by publication bias, there will be no deviations from the review protocol based on the findings from the included articles. However, when an amendment is very necessary and justifiable, such amendment(s) will be reported and implemented. We will report any amendments made especially regarding research questions during the publication of the review.

\section{Discussion}

Individuals living with these underlying conditions such as diabetes, heart diseases, hypertension, cardiocerebrovascular disease and cancer, are prone to developing and dying from COVID-19. One possible reason is that individuals with these conditions have compromised immunity which makes them susceptible to COVID-19. While researchers are developing vaccines and treatments for the COVID-19, 
prevention strategies are the best available options. Exercise has proven to increase the immunity of individuals with these proxy conditions that makes them susceptible to COVID-19. While reviews on the impact of exercise on the immune parameters on these proxy conditions exist sparingly, there is no comprehensive systematic and meta-synthetic evidence to determine FITT parameters for use by researchers who are developing RCTs specific for decreasing the risk and slowing down the impact of COVID-19. Therefore, the need for this review. Furthermore, this study will provide useful information, especially concerning the current public health emergency, for clinical practice, future research related to this topic and also inform government on health care decision making at the community, national, and international levels.

\section{Abbreviations}

\section{CD4}

Cluster of differentiation 4

CD8

Cluster of differentiation 4

COPD

Chronic obstructive pulmonary disease

\section{COVID-19}

Coronavirus disease of 2019

CRP

C-reactive protein

DM

Diabetes mellitus

FITT

Frequency, Intensity, Time, Type

\section{GRADE}

Grading of recommendation, assessment, development and evaluation

\section{$\lg A$}

Immunoglobulin-A

\section{$\lg D$}

Immunoglobulin-D

IgE

Immunoglobulin-E

IgG

Immunoglobulin-G

\section{$\lg \mathrm{M}$}

Immunoglobulin-M

IL-10

Interleukin-10 
IL-1 $\beta$

Interleukin-1Beta

IL-6

Interleukin-6

PICO

Population, Intervention, Comparison, Outcome

PRISMA-P

Preferred Reporting Items for systematic reviews and Meta-Analyses Protocol PROSPERO

International Prospective Register of Systematic Reviews

RCT

Randomized controlled trial

\section{REV MAN}

Review manager

ROB

Risk of bias

SARS-CoV-2

severe acute respiratory syndrome coronavirus

T cells

Thymus cells

TNF-a

Tumor necrosis factor-a

WHO

World Health Organization

\section{Declarations}

\section{Ethics approval and consent to participate}

Not applicable

Consent for publication

Not applicable

\section{Availability of data and materials}

Not applicable

\section{Competing interests}

The authors declare that they have no competing interests. 


\section{Funding}

No funding was gotten for the study. However, the principal investigator, Ebuka Miracle Anieto, is a recipient of the Mandela Rhodes Scholarship which provided him full sponsorship for his master's degree programme.

\section{Authors' contributions}

EMA, VEO, and OJA conceived the protocol. EMA, VEO, IBN, BCMS, JM and MEK designed the protocol. EMA, VEO, IBN, MEK developed the search strategy. All the authors contributed to the reading, editing and approving of the final draft of the manuscript.

\section{Acknowledgements}

Not applicable

\section{References}

1. World Health Organization. WHO Director-General's Remarks at the Media Briefing on 2019-nCoV. 2020.

2. Wuhan Municipal Health Commission. Report of clustering pneumonia of unknown etiology in Wuhan City. 2019.

3. Kang S, Peng W, Zhu Y, Lu S, Zhou M, Lin W, Wu W, Huang S, Jiang L, Luo X, Deng M. Recent Progress in understanding 2019 novel coronavirus associated with human respiratory disease: detection, mechanism and treatment. Int J Antimicrob Agents. 2020;29:105950.

4. World Health Organization. WHO coronavirus disease (COVID-19) dashboard. 2020.

5. American Thoracic Society. New study identifies characteristics of patients with fatal COVID-19. 2020.

6. Carlos WG, Dela Cruz CS, Cao B, Pasnick S, Jamil S. COVID-19 Disease due to SARS-CoV-2 (Novel Coronavirus). Am J Respir Crit Care Med. 2020;15(4):7-8. 201 (.

7. Chen N, Zhou M, Dong X, Qu J, Gong F, Han Y, Qiu Y, Wang J, Liu Y, Wei Y, Yu T. Epidemiological and clinical characteristics of 99 cases of 2019 novel coronavirus pneumonia in Wuhan, China: a descriptive study. The Lancet. 2020;15(395(10223):507-13.

8. Zhou Y, Yang L, Han M, Huang M, Sun X, Zhen W, et al. Clinical reports on early diagnosis of novel coronavirus (2019-nCoV) pneumonia in stealth infected patients. Preprints 2020:2020020156.

9. Liu Y, Yang Y, Zhang C, Huang F, Wang F, Yuan J, et al. Clinical and biochemical indexes from 2019nCoV infected patients linked to viral loads and lung injury. Sci China Life Sci. 2020;63:364-74.

10. Li T, Wei C, Li W, Hongwei F, Shi J. Beijing Union Medical College Hospital on" pneumonia of novel coronavirus infection" diagnosis and treatment proposal (V2. 0). Med J Peking Union Med Coll Hosp. 2020. 
11. Li B, Yang J, Zhao F, Zhi L, Wang X, Liu L. Prevalence and impact of cardiovascular metabolic diseases on COVID-19 in China. Clin Res Cardiol. 2020.

12. Zheng Z, Peng F, Xu B, Zhao J, Liu H, Peng J, Li Q, Jiang C, Zhou Y, Liu S, Ye C. Risk factors of critical \& mortal COVID-19 cases: A systematic literature review and meta-analysis. Journal of Infection. 2020.

13. Parohan $M$, Yaghoubi S, Seraji A, Javanbakht MH, Sarraf P, Djalali M. Risk factors for mortality in patients with Coronavirus disease 2019 (COVID-19) infection: a systematic review and meta-analysis of observational studies. The Aging Male. 2020;5:1-9.

14. Tian W, Jiang W, Yao J, Nicholson CJ, Li RH, Sigurslid HH, Wooster L, Rotter JI, Guo X, Malhotra R. Predictors of mortality in hospitalized COVID-19 patients: A systematic review and meta-analysis. Journal of Medical Virology. 2020.

15. Jain V, Yuan JM. Systematic review and meta-analysis of predictive symptoms and comorbidities for severe COVID-19 infection. medRxiv. 2020.

16. WHO. COVID-19: Vulnerable and high risk groups. 2020.

17. Bavishi C, Maddox TM, Messerli FH. Coronavirus disease 2019 (COVID-19) infection and renin angiotensin system blockers. JAMA cardiology. 2020.

18. Dana TG, Rayyan AK. Diabetic complications and dysregulated innate immunity. Front Biosci. 2008;13:1227-39.

19. Jafar N, Edriss $H$, Nugent $K$. The effect of short-term hyperglycemia on the innate immune system. Am J Med Sci. 2016;1(2):201-11. 351(.

20. Juliana C, Janine C, Cresio A. Infections in patients with diabetes mellitus: a review of pathogenesis. Indian J Endocrinol Metab. 2012;16:27-36.

21. Savoia C, Schiffrin EL. Vascular inflammation in hypertension and diabetes: molecular mechanisms and therapeutic interventions. Clin Sci. 2007;1(7):375-84. 112(.

22. Brandacher G, Winkler C, Schroecksnadel K, Margreiter R, Fuchs D. Antitumoral activity of interferon- $\gamma$ involved in impaired immune function in cancer patients. Current drug metabolism. 2006; 1;7(6):599-612.

23. Xu Z, Shi L, Wang Y, Zhang J, Huang L, Zhang C, Liu S, Zhao P, Liu H, Zhu L, Tai Y. Pathological findings of COVID-19 associated with acute respiratory distress syndrome. The Lancet respiratory medicine. 2020; 1;8(4):420-2.

24. Zhang B, Zhou X, Qiu Y, Feng F, Feng J, Jia Y, Zhu H, Hu K, Liu J, Liu Z, Wang S. Clinical characteristics of 82 death cases with COVID-19. MedRxiv. 2020.

25. Diao B, Wang C, Tan Y, Chen X, Liu Y, Ning L, Chen L, Li M, Liu Y, Wang G, Yuan Z. Reduction and functional exhaustion of T cells in patients with coronavirus disease 2019 (COVID-19). Front Immunol. 2020;1:11:827.

26. Huang C, Wang Y, Li X, Ren L, Zhao J, Hu Y, et al. Clinical features of patients infected with 2019 novel coronavirus in Wuhan, China. Lancet. 2020;395:497-506. 
27. Wang D, Hu B, Hu C, Zhu F, Liu X, Zhang J, et al. Clinical characteristics of 138 hospitalized patients with 2019 novel coronavirus-infected pneumonia in Wuhan, China. JAMA. 2020; e201585.

28. Blake JG, Ridker PM. Novel clinical markers of vascular wall inflammation. Circ Res. 2001;89:76371.

29. Blake GJ, Rifai N, Buring JE, Ridker PM. Blood pressure, Creactive protein, and risk of future cardiovascular events. Circulation. 2003;108:2993-9.

30. Fernandez-Real JM, Ricart W. Insulin resistance and chronic cardiovascular inflammatory syndrome. Endocr Rev. 2003;24:278-301.

31. Gan WQ, Man SF, Senthilselvan A, Sin DD. Association between chronic obstructive pulmonary disease and systemic inflammation: a systematic review and a meta-analysis. Thorax. 2004;1(7):59. $574-80$.

32. Pradhan AD, Manson JE, Rifai N, Buring JE, Ridker PM. Creactive protein, interleukin-6 and risk of developing type 2 diabetes mellitus. JAMA. 2001;286:327-34.

33. Preston RA, Ledford M, Materson BJ, Baltodano NM, Memon A, Alonso A. Effects of severe, uncontrolled hypertension on endothelial activation: soluble vascular cell adhesion molecule-1, soluble intercellular adhesion molecule-1 and von Willebrand factor. J Hypertens. 2002;20:871-7.

34. Sesso HD, Buring JE, Rifai N, Blake GJ, Gaziano JM, Ridker P. M. C-reactive protein and the risk of developing hypertension. JAMA J Am Med Assoc. 2003;290:2945-51.

35. Jablonska E, Piotrowski L, Grabowska Z. Serum Levels of IL-I $\beta$, IL-6, TNF-a, sTNF-RI and CRP in Patients with oral cavity cancer. Pathology \& Oncology Research. 1997; 1;3(2):126-9.

36. Zhang C, Wu Z, Li JW, Zhao H, Wang GQ. The cytokine release syndrome (CRS) of severe COVID-19 and Interleukin-6 receptor (IL-6R) antagonist Tocilizumab may be the key to reduce the mortality. Int $\mathrm{J}$ Antimicrob Agents. 2020;29:105954.

37. Brune K, Frank J, Schwingshackl A, et al. Pulmonary epithelial barrier function: some new players and mechanisms. Am J Physiol Lung Cell Mol Physiol. 2015;308(8):731-45.

38. Knudsen $L$, Ochs $M$. The micromechanics of lung alveoli: structure and function of surfactant and tissue components. Histochem Cell Biol. 2018;150(6):661-76.

39. Leiva-Juarez MM, Kolls JK, Evans SE. Lung epithelial cells: therapeutically inducible effectors of antimicrobial defense. Mucosal Immunol. 2018;11(1):21-34.

40. Simpson RJ, Kunz H, Agha N, Graff R. Exercise and the regulation of immune functions. Prog Mol Biol Transl Sci. 2015;135:355-80.

41. Adams GR, Zaldivar FP, Nance DM, Kodesh E, Radom-Aizik S, Cooper DM. Exercise and leukocyte interchange among central circulation, lung, spleen, and muscle. Brain Behav Immun. 2011;25:65866.

42. Bigley AB, Rezvani K, Chew C, Sekine T, Pistillo M, Crucian B, et al. Acute exercise preferentially redeploys NK-cells with a highly-differentiated phenotype and augments cytotoxicity against lymphoma and multiple myeloma target cells. Brain Behav Immun. 2014;39:160-71. 
43. Gupta P, Bigley AB, Markofski M, Laughlin M, LaVoy EC. Autologous serum collected $1 \mathrm{~h}$ postexercise enhances natural killer cell cytotoxicity. Brain Behav Immun. 2018;71:81-92.

44. Nieman DC, Henson DA, Austin MD, Brown VA. Immune response to a 30-minute walk. Med Sci Sports Exerc. 2005;37:57-62.

45. Church TS, Barlow CE, Earnest CP, Kampert JB, Priest EL, Blair SN. Associations between cardiorespiratory fitness and C-reactive protein in men. Arterioscler Thromb Vasc Biol. 2002;22:1869-76.

46. Esposito K, Pontillo A, Di Palo C, Gingliano G, Masella M, Marfella R. Guigliano, D. Effect of weight loss and lifestyle changes on vascular inflammatory markers in obese women. JAMA. 2003;289:1799-804.

47. Goldhammer E, Tanchilevitch A, Maor I, Beniamini Y, Rosenchein U, Sagiv M. Exercise training modulates cytokines activity in coronary heart disease patients. Int J Cardiol. 2005;100:93-9.

48. Kohut ML, McCann DA, Russell DW, Konopka DN, Cunnick JE, Franke WD, Castillo MC, Reighard AE, Vanderah E. Aerobic exercise, but not flexibility/resistance exercise, reduces serum IL-18, CRP, and IL6 independent of $\beta$-blockers, BMI, and psychosocial factors in older adults. Brain Behav Immun. 2006;20(3):201-9.

49. Smith JK, Dykes R, Douglas JE, Krishnaswamy G, Berk S. Long-term exercise and atherogenic activity of blood mononuclear cells in persons at risk of developing ischemic heart disease. JAMA. 1999;281:1722-7.

50. You T, Berman DM, Ryan AS, Nicklas BJ. EVects of hypocaloric diet and exercise training on inXammation and adipocyte lipolysis in obese postmenopausal women. J Clin Endocrinol Metab. 2004;89:1739-46.

51. Hopps E, Canino B, Caimi G. Effects of exercise on inflammation markers in type 2 diabetic subjects. Acta diabetologica. 2011;48(3):183-9.

52. Lamina S, Okoye GC. Effect of interval exercise training programme on C-reactive protein in the nonpharmacological management of hypertension: a randomized controlled trial. Afr J Med Med Sci. 2012;41(4):379-86.

53. Hagstrom AD, Marshall PW, Lonsdale C, Papalia S, Cheema BS, Toben C, Baune BT, Singh MA, Green $S$. The effect of resistance training on markers of immune function and inflammation in previously sedentary women recovering from breast cancer: a randomized controlled trial. Breast cancer research treatment. 2016;155(3):471-82.

54. Moher D, Shamseer L, Clarke M, et al. Preferred reporting items for systematic review and metaanalysis protocols (PRISMA-P) 2015 statement. Syst Rev. 2015;4:1.

55. Shamseer L, Moher D, Clarke M, Ghersi D, Liberati A, Petticrew M, Shekelle P, Stewart LA. Preferred reporting items for systematic review and meta-analysis protocols (PRISMA-P) 2015: elaboration and explanation. Bmj. 2015;2:349:g7647.

56. World Health Organization. Physical activity. 2018. 
57. Higgins J, Green S. Cochrane handbook for systematic reviews of interventions Version 5.1.0[updated March 2011].The Cochrane Collaboration, 2011.

58. Akers J, Aguiar-lbáñez R, Baba-Akbari Sari A. CRD's guidance for undertaking reviews in health care. York: Centre for Reviews and Dissemination (CRD); 2009.

59. Sterne JA, Savović J, Page MJ, Elbers RG, Blencowe NS, Boutron I, Cates CJ, Cheng HY, Corbett MS, Eldridge SM, Emberson JR. RoB 2: a revised tool for assessing risk of bias in randomised trials. bmj. 2019;28;366.

60. Guyatt GH, Oxman AD, Vist GE, et al. GRADE: an emerging consensus on rating quality of evidence and strength of recommendations. BMJ. 2008;336:924-6.

61. Cochrane Consumers and Communication Review Group. Communication review group: Data extraction template for Cochrane reviews. Cochrane Collaboration, 2007 (Updated Dec 2016).

62. Pluye P, Hong QN. Combining the power of stories and the power of numbers: mixed methods research and mixed studies reviews. Pub Health. 2014;35:29.

63. Egger M, Davey Smith G, Schneider M, et al. Bias in meta-analysis detected by a simple, graphical test. BMJ. 1997;315:629-34.

\section{Supplementary Files}

This is a list of supplementary files associated with this preprint. Click to download.

- AppendicesBMCSRSubmit.docx

- PRISMAPchecklistBMCSRSubmit.docx 